\title{
Europäischer Gerichtshof für Menschenrechte und anonyme Geburten in Frankreich
}

Ein Urteil des Europäischen Gerichtshofs für Menschenrechte in Straßburg vom 13. 2. 2003 hat auch in Deutschland ein vielfältiges Echo gefunden. ${ }^{1}$ Die in Paris lebende Französin Pascale Odièvre wollte wissen, wer ibre leibliche Mutter ist. Das Gericht in Frankreich hat den Antrag abgelehnt. Der von ibr gegen diese Entscheidung angerufene Gerichtshof für Menschenrechte in Straßburg hat ibr nicht geholfen.

\section{Die Geschichte Pascale Odièvres}

Pascale Odièvre ist heute 37 Jahre alt. Sie ist eine von inzwischen 400.000 Französinnen und Franzosen, die anonym geboren oder ausgesetzt wurden und ihre leiblichen Eltern nie kennen gelernt haben. ${ }^{2}$ Ihre Mutter hat sie im Jahr 1965 in Paris - als wohl zweites Kind - entbunden und nach der Geburt anonym zurückgelassen. Pascale Odièvre lebte daraufhin zunächst als »Mündel des Staates«, elternlos mit Hilfe der »DASS «, der französischen Einrichtung, die sich in diesen Fällen der Kinder annimmt. Sie wurde mit noch nicht ganz 4 Jahren von dem Ehepaar Odièvre adoptiert. ${ }^{3}$ I990 beantragte Pascale Odièvre Einsicht in ihre Akten. Sie erhielt lediglich die vorhandenen Informationen, die nicht dem Schutz der Anonymität der Mutter unterlagen; Odièvre erfuhr also nicht die Identität ihrer leiblichen Eltern. Dieses »bulletin de renseignement«, eine amtliche Auskunft, berichtete ihr über die Umstände, unter denen ihre Mutter sie entbunden und dann dem Staat übergeben hatte.

Aus den ihr überlassenen Unterlagen konnte Odièvre entnehmen, dass ihre leiblichen Eltern vor ihrer Geburt seit etwa sieben Jahren in einer eheähnlichen Gemeinschaft gelebt hatten. Vor ihr hatte die Mutter vom gleichen Mann einen Sohn geboren; der Vater, ein Spanier, der in Frankreich als Anstreicher arbeitete, war mit einer anderen Frau verheiratet. ${ }^{4}$

Odièvres Mutter hatte I 965 angegeben, der Vater wolle von Pascale »nichts wissen«, könne die Last eines weiteren Kindes nicht tragen, verdiene gerade I.200,00 Franc (ca.200,00€) im Monat; die Familie sei außerdem von der Zwangsräumung bedroht.

I An dieser Stelle herzlichen Dank an Prof. Dr. Alfred Wolf für die zahlreichen Anregungen und die engagierten Diskussionen, die zu der Entstehung dieses Artikels geführt haben.

Europäischer Gerichtshof für Menschenrechte, Entscheidung vom I3. 2. 2003, no. 42326/98 (Odièvre v. France) - im Folgenden: EGMR, I3. 2. 2003.

Das Urteil ist veröffentlicht unter: http://www.echr.coe.int/Fr/Judgments.htm. Inzwischen liegt eine Übersetzung des Urteils von Meyer-Ladewig, Wachtberg, Petzold vor: NJW 2003, S. 2 I 45 ff.

2 Vgl. Internetseite der französischen Regierung: http://www.service-public.fr/accueil/accouchementX.html

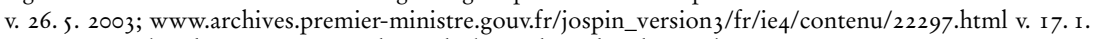
2003; Swientek, Christine, Die Wiederentdeckung der Schande, Freiburg 2001, S. I 82.

3 DASS ist die Abkürzung für »direction de l'action sanitaire et sociale « zuständig ist der Service de l'Aide Sociale à l'Enfance, vgl. Art. 63 code de la famille et de l'aide sociale à l'enfance (C.fam.).

4 EGMR, I3. 2. 2003 (Fn. I), \I2. 
Die zuständigen Sozialarbeiter beschrieben Odièvres Mutter als willenlos, den

Wünschen und Bedürfnissen des Kindesvaters erlegen. Eine Frau, die ohne weitere Skrupel oder moralische Zwänge die Tochter zurücklasse.5 Die Mutter hat in den darauf folgenden Jahren noch zwei Söhne bekommen. ${ }^{6}$

\section{Das Verfabren in Frankreich}

Pascale Odièvre forderte von den französischen Behörden die Überlassung auch der Informationen aus ihren Akten, die Aufschluss über die Identität der leiblichen Familie geben. ${ }^{7}$

Ihre 1998 vor dem » Tribunal de Grande Instance « von Paris erhobene Klage gegen den französischen Staat auf Offenbarung des Namens ihrer leiblichen Mutter wurde abgewiesen. Daraufhin rief Pascale Odièvre im selben Jahr den Europäischen Gerichtshof an, der trotz fehlender Rechtswegerschöpfung die Sache zur Entscheidung annahm. ${ }^{8}$

\section{Die anonyme Geburt in Frankreich}

\section{Die Entwicklung bis zum I8. Jabrbundert}

In Frankreich hat die intensive Beschäftigung mit dem Problem der Kindesaussetzung eine lange Tradition.

Bereits im 17. Jahrhundert wurden Drehtüren (tourniquet) in der Außenmauer von Findelhäusern angebracht. ${ }^{9}$ Eltern konnten ihr Kind in diese Tür legen und eine Klingel betätigen. Ein Mechanismus drehte die Tür und beförderte das Kind ins Innere des Hauses, wo es versorgt werden konnte. ${ }^{10}$ Drehtüren waren nach damaliger Vorstellung ein Akt christlicher Nächstenliebe. Sie sollten darüber hinaus den Familienfrieden wahren, indem die Frau die Frucht eines ehelichen Fehltritts verheimlichen und der leibliche Vater sich drohender (Unterhalts-)Verpflichtungen entziehen konnte. ${ }^{11}$ Nach Schätzungen soll es im Jahre 1780 ca. 250 Drehtüren gegeben haben, in die bis zu $\mathrm{I} 30.000$ Kinder jährlich gelegt worden sind. ${ }^{12}$

Einrichtungen wie die Drehtüren gab es auch in anderen, fast immer katholisch geprägten Gebieten Europas. Klöster haben seit dem Mittelalter Findelkinder aufgenommen und aufgezogen. Deren Schutz und Fürsorge zählte zu den karitativen Aufgaben der Kirche. In protestantischen Gebieten Europas wurden Drehtüren nicht eingeführt, sondern abgelehnt. ${ }^{13}$

5 EGMR, I3. 2. 2003 (Fn. I), \I 2.

6 EGMR, I3. 2. 2003 (Fn. I), $\mathbb{S}$ I3.

7 EGMR, I3. 2. 2003 (Fn. I), \ 12.

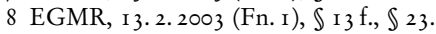

9 Benda, Die »anonyme« Geburt, JZ 2003, 533 (535); vgl. auch Frank, Rainer/Helms, Tobias, Rechtliche Aspekte der anonymen Kindesabgabe in Deutschland und Frankreich, FamRZ 200I, S. I 340 ff. (I 343 ).

I० EGMR, I 3. 2. 2003 (Fn. I), \ I5; vgl. auch Helms, Die Feststellung der biologischen Abstammung, Berlin I 999 , S. I4.

i I Helms (Fn. Io), S. I4.

I 2 Frank/Helms, FamRZ 200 , S. I343; Helms (Fn. Io), S. I 4 f.

I 3 Hunecke, Volker, Die Findelkinder von Mailand, Stuttgart I 987 , S. I 4 ff.; 52 ff. (eingehend zur italienischen Geschichte der Findelfürsorge). 
Im Zuge der Aufklärung wurde die Zweckmäßigkeit von Findelhäusern und Einrichtungen wie die der Drehtüren kontrovers diskutiert. Man erkannte, dass die anonyme Kindesaussetzung kein geeignetes Mittel war, um Kindestötung zu verhindern, und nahm an, dass die Möglichkeit der anonymen Kindesabgabe geradezu Anreiz zur Kindesaussetzung bot. ${ }^{14}$

In der zweiten Hälfte des i9. Jahrhunderts schaffte man in Frankreich die Drehtüren nach und nach ab und ersetzte sie durch »bureaux ouverts «, Tag und Nacht geöffnete staatliche Stellen, in denen Eltern diskret, aber zunächst nicht anonym, ihre Kinder abgeben konnten. ${ }^{\text {Is }}$

Im I9. Jahrhundert endete also die Geschichte der »Drehtüren«, der »französischen Babyklappen«. Frankreich hat seitdem nicht über ihre Wiedereinrichtung nachgedacht.

\section{Die weitere Entwicklung bis $194 I$}

Mit Gesetz vom 27.6. $1904^{16}$ ermöglichte Frankreich wieder, Kinder anonym in den »bureaux ouverts« zurückzulassen. Grund für diese neuerliche Wende könnten demographische Veränderungen am Ende des i9. Jahrhunderts gewesen sein. Die ersten Verhütungsmethoden und die Folgen des deutsch-französischen Krieges sorgten für einen unerwünschten Geburtenrückgang. ${ }^{17}$ Man wollte die anonymen Kinder wieder gewinnen. Dennoch: Eine Rückkehr zu den »Drehtüren « des I7. Jahrhunderts gab es nicht.

\section{Die neueren französischen Regelungen zur anonymen Geburt}

\section{Die Entwicklung von 194 I bis 1993}

Die Vichy-Regierung hat mit Gesetzesdekret vom 2.9. I94 I »über den Schutz der Geburt « die anonyme Geburt neu geregelt.

Grund dieser neuen Regelung war nicht in erster Linie die Sorge um die Kinder. Im Vordergrund stand die Wahrung des Familienfriedens: Französische Ehefrauen sollten die Kinder, die aus Beziehungen mit deutschen Soldaten hervorgegangen waren, ohne größere Schwierigkeiten entbinden und abgeben können. ${ }^{18}$ Den schwangeren Frauen wurde die Wahrung des Geheimnisses ihrer Identität garantiert und ermöglicht, auf Kosten des Staates einen Monat vor sowie einen Monat nach der Geburt im Krankenhaus oder in speziell für Frauen in Not eingerichteten Häusern, so genannten »maisons maternelles «, versorgt $\mathrm{zu}$ werden. ${ }^{19}$

I4 Vgl. Frank/Helms, FamRZ 200I, S. I344; Helms (Fn. I0), S. I 5 , der den Grund in der aufkommenden Kritik an der Kindesaussetzung mittels Drehtüren in der größeren, sexuellen Freizügigkeit, der Verarmung "unterer" Schichten sowie dem damit verbundenen Anstieg der anonymen Kindesabgaben sieht.

is EGMR, I3. 2. 2003 (Fn. I), \ Is; Helms (Fn. I0), S. I s, der unter anderem ermittelt, dass es im Jahr i 860 in Frankreich nur noch 25 Drehtüren gab; Scheiwe, Babyklappe und anonyme Geburt, ZRP 200I, 368 (369).

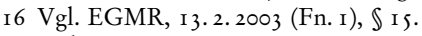

I7 Helms (Fn. Io), S. I 5 .

I 8 Vgl. Dourgnon/Egéa, L'accouchement sous X-un demi siècle après la loi Pétain, http://perso.wanadoo.fr/ amo-buron/aprespetain.htm v. I7. I. 03; Swientek (Fn. 2), S. I 8 I; vgl. aber auch Helms (Fn. I0), S. I6, der den Grund für die neue Regelung der Vichy-Regierung zunächst in dem erneuten, kriegsbedingten Geburtenrückgang sieht.

I9 EGMR, I3. 2. 2003 (Fn. I), \Is. 
Mit Gesetz vom 8. I. I $993^{20}$ wurde dieses Recht auf Anonymität in das französische Zivilgesetzbuch übernommen. Art. 34I-I des Code Civil (C. C.) lautet noch heute: »Anlässlich der Niederkunft kann die Mutter verlangen, dass das Geheimnis ihrer Erklärung und Identität gewahrt wird. $\ll^{21}$ Nach französischem Recht ist die nichteheliche Mutter mit ihrem Kind im juristischen Sinne nicht automatisch verwandt. Die Regel »mater semper certa est « findet in Frankreich keine Anwendung. ${ }^{22}$ Die rechtliche Abstammung zum nichtehelichen Kind wird nur begründet, wenn die Mutter das Kind anerkennt. ${ }^{23}$ Verweigert die Mutter die Anerkennung, werden in der Geburtsurkunde keine weiteren Angaben zu den Eltern gemacht. Art. 57 C. C. lautet: »Werden der Vater und die Mutter eines unehelichen Kindes oder wird einer von ihnen nicht angegeben, so darf dieser (der Zivilstandsbeamte, Fn.d. A.) keine Angabe darüber in den Registern machen. $\ll^{24}$

Die neuen Vorschriften mit Gesetz vom 8. I. I 993 erschwerten außerdem die Suche nach der leiblichen Mutter und die Möglichkeit einer Mutterschaftsklage. Während bis zum Januar 1993 nach der alten Fassung des Art. 34 I C. C. jedes Kind bei Vorliegen schwerwiegender Vermutungen (»indices graves«), dass es von einer bestimmten Frau geboren worden sei, die Möglichkeit hatte, Mutterschaftsklage zu erheben, ist dies anonym geborenen Kindern nach der neuen und heute noch geltenden Regelung des Art. 34I C. C. verwehrt.

Art. 34 I C. C. bestimmt, dass die Mutterschaftsklage immer dann nicht statthaft ist, wenn die Entbindung anonym im Sinne des Art. 34 I-I C. C. erfolgt ist. Der Frau, die von der Möglichkeit der anonymen Geburt Gebrauch macht, wird also eine lebenslange Anonymität zugesichert.

Insbesondere seit der Gesetzesreform aus dem Jahr I993 war die Regelung zur anonymen Geburt in Frankreich heftig umstritten. Frankreich verzeichnet derzeit ca. 600 anonyme Geburten im Jahr. ${ }^{25}$ Inzwischen haben ca. 400.000 Menschen in Frankreich keine Kenntnis über ihre biologische Herkunft. Der Volksmund bezeichnet diese anonyme Geburt als »accouchement sous $\mathrm{X} «$. »Accouchement« bedeutet Geburt oder Entbindung. »Sous $\mathrm{X}$ « wird hinzugefügt, weil das anonym geborene Kind im Geburtsregister in Ermangelung leiblicher Eltern, die das Kind anerkennen, unter einer besonderen Kategorie, der Kategorie »X $\mathrm{X}$ eingetragen wird. Ein Begriff, den heute auch die juristische Fachliteratur weitgehend übernommen hat.

Es mehrten sich seit Beginn der 9oer Jahre Forderungen von Betroffenen, Soziologen, Ärzten und Juristen, den anonym geborenen Menschen bessere rechtliche und tatsächliche Möglichkeiten an die Hand zu geben, als Erwachsene die Identität ihrer biologischen Eltern ermitteln zu können. ${ }^{26}$ Im Vordergrund der öffentlichen Diskussion standen nunmehr die Rechte des Kindes, der Schutz seiner Identität und seine Persönlichkeitsentfaltung sowie das Recht auf Kenntnis der eigenen Herkunft.

20 Gesetz Nr.93-22 vom 8. I. I993, vgl. EGMR, I3.2. 2003 (Fn. I), \Is.

2 I Art. 34I-I C. C., Übersetzung nach Bergmann/Ferid, Internationales Ehe- und Kindschaftsrecht, Frankfurt a. M. 1999, Stand: I42. Lieferung, 4. 200I, Frankreich, S. 95 .

22 EGMR, I3. 2. 2003 (Fn. I), \I5.

23 Art. 334-8, Absatz I C. C.: »La filiation naturelle est légalement établie par reconnaissance volontaire«.

24 »Si les père et mère de l'enfant naturel, ou l'un d'eux, ne sont pas désignés à l'officier de l'état civil, il ne sera fait sur les registres aucune mention à ce sujet «, Übersetzung nach Bergmann/Ferid (Fn. 2I), S. 60.

25 EGMR, I3. 2. 2003 (Fn. I), \36; vgl. Frank/Helms, FamRZ 200 I S. I 345 m.w.N; Internetseite der französischen Regierung: www.social.gouv.fr/famille-enfance/doss_pr/34_00 I 2 I 4.htm v. I7. I. 03 .

26 Z.B. http://www.x-en-colere.org/php/No v. I7. I.03; vgl. auch Scheiwe, Babyklappe und anonyme Geburt, ZRP 200I, 368 (370); s.a. http://www.senat.fr/lc/lc70/lc700.html; http://tempo2.phpnet.org/ dorigine/index.html v. I7. I. 2003 . 
Daraufhin wurde im Jahre 2002 ein neues Amt eingerichtet, der »Conseil national pour l'accès aux origines personelles «, kurz: CNAOP. Seine Aufgaben sind im französischen Sozial- und Familiengesetz geregelt. ${ }^{27}$ Der CNAOP sammelt nach jeder anonymen Geburt alle Informationen und Anfragen der Beteiligten. Liegen zwei übereinstimmende Anfragen vor - möchte also das Kind die Identität der leiblichen Eltern erfahren und ist die Mutter damit einverstanden - leitet der CNAOP die erforderlichen Angaben an die Auskunftssuchenden weiter. ${ }^{28}$ Ist die leibliche Mutter zum Zeitpunkt des Auskunftsverlangens des Kindes bereits gestorben, hat das Kind einen Anspruch, ihre Identität zu erfahren, es sei denn, die Mutter hat vor ihrem Tod ausdrücklich das Gegenteil verfügt. ${ }^{29}$

Art. 222-6 des »code de l'action sociale et des familles « sieht vor, dass die Mutter bei der Geburt über die Möglichkeit der späteren Aufhebung ihrer Anonymität aufgeklärt wird. Eine Aufklärung der Mutter findet außerdem im Hinblick auf mögliche psychische Belastungen statt, denen Kinder ausgesetzt sein können, wenn ihnen ihre biologische Herkunft dauerhaft unbekannt bleibt. Die Mutter wird gebeten, ${ }^{\circ}$ Informationen über die näheren Umstände der Geburt, über den Vater und die gesundheitlichen Merkmale der leiblichen Eltern zu hinterlassen. In einem verschlossenen Umschlag kann sie Angaben zu ihrer Person hinterlegen, die ihre spätere Identifizierung ermöglichen. ${ }^{3}$ Diese neue französische Regelung lässt zwar die Möglichkeit der anonymen Geburt weiter bestehen; sie geht jedoch einen Schritt zur Transparenz im Interesse anonym geborener Kinder.

Dennoch: Pascale Odièvre hat auch nach dieser neuen Regelung aus dem Jahr 2002 keinen Anspruch darauf, die Identität ihrer leiblichen Verwandten gegen deren Willen zu erfahren.

\section{Verfabrensrechtliche Aufgaben des Europäischen Gerichtshofs für Menschenrechte}

Sie hatte deshalb gegen die Entscheidung in Frankreich Individualbeschwerde beim Europäischen Gerichtshof für Menschenrechte in Straßburg erhoben. Nach Art. 34 EMRK kann der Gerichtshof von jeder natürlichen Person mit der Begründung angerufen werden, durch einen Mitgliedsstaat in einem Recht aus der Konvention zum Schutze der Menschenrechte und Grundfreiheiten verletzt zu sein. ${ }^{32}$

Bei den in der EMRK verbürgten Menschenrechten handelt es sich zunächst um klassische Abwehrrechte des Bürgers gegen den Staat. ${ }^{33}$ Die einzelnen Rechte können

27 Vgl. Art. I 47-I code de l'action sociale et des familles. (C.act.soc.), eingeführt durch das Reformgesetz vom Januar 2002, Gesetz Nr. 2002-93 vom 22. I. 2002 « relative à l'accès aux origines des personnes adoptées et pupilles de l'État «.

28 Die genauen Voraussetzungen für Weitergabe der Informationen sind in Art. I 47-2 ff. C.act. soc. geregelt und im Internet abrufbar unter: http://www.adminet.com/jo/20020I23/MESX02053 I8L.html v. I4. 2. 2003.

29 Vgl. Art. I47-6 C. act. soc.

30 Art. 222-6, Absatz I C. act. soc.: »(...). Elle est donc invitée à laisser...«

3 I Art. 222-6 Absatz I C. act. soc.; vgl. auch EGMR, I3.2. 2003 (Fn. I), \$I7.

32 Art. 34 ERMK lautet: »Der Gerichtshof kann von jeder natürlichen Person, nichtstaatlichen Organisation oder Personengruppe, die behauptet, durch eine der Hohen Vertragsparteien in einem der in dieser Konvention oder den Protokollen dazu anerkannten Rechten verletzt zu sein, mit einer Beschwerde befasst werden. Die Hohen Vertragsparteien verpflichten sich, die wirksame Ausübung dieses Rechts nicht zu behindern.«; vgl. auch EMRK, Handkommentar (im Folgenden: Hk-EMRK), Baden-Baden 2003, Art. 34, Rn. 4.

33 Villiger, Mark E., Handbuch der Europäischen Menschenrechtskonvention, Zürich I999, Rn. 23 I; Binder, 
jedoch auch Schutzpflichten des Staates begründen. Art. 8 bis I I der EMRK garantieren in ihren jeweils ersten Absätzen einzelne Grundrechte, während der zweite Absatz der jeweiligen Bestimmungen Eingriffsmöglichkeiten, Rechtfertigungsgründe und Schranken bestimmen. Aufgrund dieser parallelen Struktur der in der EMRK verbürgten Menschenrechte hat der Gerichtshof für die Prüfung einer Rechtsverletzung aus Art. 8-I I EMRK einheitliche Kriterien und Auslegungsregeln entwickelt, denen das Straßburger Gericht auch in der Entscheidung über die Individualbeschwerde Odièvres gefolgt ist.

Anhand einer rechtsvergleichenden Betrachtung des streitigen Problems stellt der Gerichtshof auch im Fall Odièvre zunächst fest, welche Regelungen andere Konventionsstaaten getroffen haben, und prüft, ob sich zu dem Problem bereits eine einheitliche, europäische Rechtsauffassung herausgebildet hat. ${ }^{34}$ Ein- und dieselbe streitige Rechtsfrage kann in verschiedenen Konventionsstaaten aufgrund unterschiedlicher historischer, religiöser oder politischer Erfahrungen zu anderen Regelungen führen. So auch für die anonyme Geburt: Während in Frankreich anonyme Geburt und anonyme Kindesabgabe seit Jahrhunderten legitim sind, kennen Staaten wie Deutschland, Spanien, Norwegen u. a. keine entsprechenden gesetzlichen Regelungen.35 Das Gericht räumt dem einzelnen Konventionsstaat bei der Wahl seiner Mittel zur Regelung einer streitigen Rechtsfrage einen weiten Beurteilungsspielraum ein. Die Lehre vom Ermessens- oder Beurteilungsspielraum (marge d'appréciation/ margin of appreciation $)^{36}$ besagt, dass zunächst der Einzelstaat für die Gewährleistung der in der EMRK verbürgten Rechte verantwortlich ist und die Kontrolle durch den Europäischen Gerichtshof nur subsidiär erfolgt.

\section{Die Entscheidung des Europäischen Gerichtshofs für Menschenrechte im Fall Odièvre}

Odièvre rügte mit ihrer Beschwerde aus dem Jahre 1998 die Verletzung ihrer Rechte aus Art. 8 EMRK. Noch vor einer Entscheidung des Gerichts im Februar 2003 war in Frankreich das neue Gesetz zur Regelung anonymer Geburten in Kraft getreten. Doch auch die neue Gesetzeslage gibt Odièvre keinen Anspruch auf Kenntnis der Identität ihrer leiblichen Verwandten. Odièvre sah darin eine fortdauernde Verletzung von Art. 8 der Menschenrechtskonvention.

\section{Zur Bedeutung von Art. 8 EMRK}

Art. 8 Abs. I EMRK gewährleistet dem Einzelnen im Wesentlichen vier Grundrechte. ${ }^{37}$ In Absatz I heißt es: »Jede Person hat das Recht auf Achtung ihres Privatund Familienlebens, ihrer Wohnung und ihrer Korrespondenz.« Odièvre rügte die Verletzung ihrer in Art. 8 EMRK geschützten Rechte des Privat- und Familienlebens.

Ulrike Elisabeth, Auswirkungen der EMRK und des UN-Übereinkommens über die Rechte des Kindes vom 20. I I. I989 auf Rechtsfragen im Bereich der medizinisch assistierten Fortpflanzung, Frankfurt a. M. I998, S. I9, 23, 33 .

34 Vgl. Binder (Fn. 33), S. 24; Urteil v. I 3. 2. 2003 (Fn. I), \I9 und $\$ 47$.

35 EGMR, I3. 2. 2003 (Fn. I), \I9f.

36 Vgl. Binder (Fn. 33), S. 26; Hk-EMRK (Fn. 32), Art. 46, Rn. 7; Villiger (Fn. 33), Rn. 553.

37 Hk-EMRK (Fn. 32), Art. 8, Rn. I. 
In einer Entscheidung aus dem Jahre $1979^{38}$ hatte der Gerichtshof den Schutzbereich des Rechts auf Wahrung des Familienlebens grundsätzlich definiert. Danach schütze Art. 8 Abs. I EMRK die bestehende Familie, gebe jedoch kein Recht auf Gründung einer Familie. 39 Die Eröffnung des Schutzbereichs von Art. 8 Abs. I EMRK setze zunächst voraus, dass ein Familienleben, geprägt durch persönliche Nähe der Familienmitglieder untereinander, tatsächlich bereits bestehe. Im Falle Odièvres bestehe die Besonderheit darin, dass zu der biologischen Familie noch gar keine Bindung existiere. Dem Schutzbereich des Rechts auf Familienleben nach Art. 8 EMRK, so bestätigte der Gerichtshof seine bisherige Rechtsprechung, unterliege daher nur das Verhältnis Odièvres zu ihrer Adoptionsfamilie, weil es sich hierbei um die tatsächlich gelebte Familienbeziehung handele. Hinsichtlich ihrer biologischen Verwandten sei der Schutzbereich des Art. 8 EMRK nicht eröffnet. $4^{\circ}$

\section{Das Recht auf Achtung des Privatlebens}

Odièvre berief sich aber auch auf die Verletzung ihres Persönlichkeitsrechts. Da sie keinen Anspruch auf Kenntnis ihrer eigenen Herkunft habe, sei sie in ihrem Persönlichkeitsrecht verletzt. In den Schutzbereich des Privatlebens falle nach der Rechtsprechung des Straßburger Gerichts unter anderem »ein Recht auf Identität und Persönlichkeitsentwicklung sowie das Recht, mit seinesgleichen und der Außenwelt Beziehungen zu knüpfen und diese weiterzuentwickeln «.4 Das Recht auf Persönlichkeitsentfaltung umfasse nach Ansicht des Gerichtshofs außerdem die Ermittlung aller Informationen über die eigene Identität sowie aller Daten und Unterlagen, die erforderlich sind, die eigene Identität, ihre Entwicklung und Geschichte oder andere wichtige Aspekte, wozu auch die Identität der Erzeuger und die näheren Umstände der Geburt zählten, zu erforschen und aufzudecken. Dieser Schutzbereich des Art. 8 Abs. I EMRK sei nach Auffassung des Europäischen Gerichtshofs berührt. Mit der neuen Regelung zur anonymen Geburt greife Frankreich in die Rechte anonym geborener Kinder aus Art. 8 EMRK ein.

\section{Rechtfertigung des Eingriffs}

Absatz 2 des Art. 8 EMRK legt die Schranken der garantierten Rechte fest: »Eine Behörde darf in die Ausübung dieses Rechts nur eingreifen, soweit der Eingriff gesetzlich vorgesehen und in einer demokratischen Gesellschaft notwendig ist für die nationale oder öffentliche Sicherheit, für das wirtschaftliche Wohl des Landes, zur Aufrechterhaltung der Ordnung, zur Verhütung von Straftaten, zum Schutz der Gesundheit oder der Moral oder zum Schutz der Rechte und Freiheiten anderer «. Im Fall Odièvres lauten die Fragen, die sich der Europäische Gerichtshof stellen musste: Berücksichtigt die französische Regelung über die anonyme Geburt aus dem Jahr 2002 in ausreichendem Maß die Interessen des Kindes sowie die Dritter und ist diese Regelung zum Schutz eines legitimen Zwecks notwendig und verhältnismäBig?

38 EGMR, Entscheidung Marckx ./. Belgien vom 13.6. I979, série A, vol. 3 I.

39 EGMR, Marckx ./. Belgien v. I 3.6.79, $\mathbb{\int} 3 \mathrm{I} f$.

$40 \mathrm{EGMR}, \mathrm{I} 3 \cdot 2.2003$ (Fn. I), $\mathbb{\$} \$ 28 \mathrm{f}$.

4I EGMR, I3.2. 2003 (Fn. I), \29: »... que l'article 8 protège un droit à l'identité et à l'épanouissement personnel et celui de nouer et de développer des relations avec ses semblables et le monde extérieur. (...) «; vgl. auch Binder (Fn. 33), S. 38 . 
Wie die geschichtliche Entwicklung der Kindesaussetzung in Frankreich gezeigt hat, wurden anonyme Geburt und Kindesabgabe immer wieder als geeignetes Mittel betrachtet, um Leben und Gesundheit von Mutter und Kind zu schützen. Beide Institute sollten sicherstellen, dass die Mutter bei der Geburt medizinische Hilfe in Anspruch nimmt und trotz einer Notlage das Kind nach der Geburt nicht aussetzt oder gar tötet. ${ }^{2}$ Der Gerichtshof stellte die Geeignetheit dieses Mittels nicht in Frage, ${ }^{43}$ obwohl nach dem Vorbringen Odièvres die Zahl der Kindesaussetzungen in Ländern, die keine anonyme Kindesabgabe kennen, ebenso hoch ist wie in Frankreich. 44

\section{Der Beurteilungsspielraum}

Der Europäische Gerichtshof erinnerte in seinem Urteil vom Februar 2003 vielmehr daran, dass der Schutz aus Art. 8 Abs. I EMRK vorrangig durch den Einzelstaat und nicht durch die Gemeinschaft gewährleistet werden muss. Der Vertragsstaat sei daher in der Wahl seiner Mittel weitgehend frei und genieße einen weiten Beurteilungsspielraum. ${ }^{45}$ Die rechtsvergleichende Auslegung des Gerichts ergebe, dass andere Mitgliedsstaaten eine mit Frankreich vergleichbare Tradition nicht kennen. ${ }^{4}$ Auch die Frage der rechtlichen Verwandtschaft eines nichtehelichen Kindes zu seiner Mutter werde in den Konventionsstaaten unterschiedlich beurteilt, so dass eine Rechtsvereinheitlichung nicht in Betracht komme und die Einzelstaaten auf den ihnen zugebilligten Beurteilungsspielraum zurückgreifen könnten. ${ }^{47}$

\section{Verbältnismäßigkeit}

Es blieb die entscheidende Frage, ob sich die französische Regelung zur anonymen Geburt aus dem Januar 2002 innerhalb dieses bestehenden Beurteilungsspielraums bewegt und ob die von der Konvention garantierten Rechte durch die nationalstaatliche Regelung ausreichend gewährleistet sind. Es ist zugleich die Frage nach der Verhältnismäßigkeit der Regelung und dem effektiven Ausgleich der widerstreitenden Interessen.

Das Recht Odièvres auf Kenntnis ihrer Abstammung stellte der Gerichtshof zunächst dem gleichberechtigten Interesse der Mutter auf Wahrung ihrer Anonymität gegenüber. ${ }^{8}$ Auch dieses Recht der Mutter sei vom Schutzbereich des Art. 8 Abs. I EMRK, dem Recht auf freie Entfaltung der Persönlichkeit, umfasst. ${ }^{49}$ Die Interessen von Mutter und Kind stellten, so der Gerichtshof, gleichwertige Rechte gem. Art. 8 Abs. I EMRK dar, die nur schwer in ein ausgewogenes Gleichgewicht zu bringen seien. In seiner Entscheidung hatte sich das Gericht im Wesentlichen von dem Argument leiten lassen, dass Odièvre volljährig ist und somit keinen besonderen Schutz Minderjähriger mehr genießen könnte. Außerdem sei die Klägerin im Alter von vier Jahren adoptiert worden und trotz der anonymen Abgabe durch die Mutter in einer intakten Familie aufgewachsen..$^{\circ}$

42 EGMR, I3. 2. 2003 (Fn. I), $\ 4$; zum Schutz des Lebens Art. 2 Abs. I S. I EMRK: »Das Recht jedes Menschen auf Leben wird gesetzlich geschützt.«

43 EGMR, I 3. 2. 2003 (Fn. I), $\$ 45$.

44 EGMR, I3. 2. 2003 (Fn. I), $\ 3$ I.

$45 \mathrm{EGMR}, \mathrm{I3}$. 2. 2003 (Fn. I), $\$ 47$.

46 EGMR, I 3. 2. 2003 (Fn. I), \47

47 EGMR, I3. 2. 2003 (Fn. I), $\$ 47$.

48 EGMR, I 3. 2. 2003 (Fn. I), $\$ 44$.

$49 \mathrm{EGMR}, \mathrm{I} 3.2 .2003$ (Fn. I), $\$ 44$.

50 EGMR, I3.2.2003 (Fn. I), $\$ 44$. 
Doch das Gericht ging noch weiter. Auch Rechte Dritter seien von dem Auskunftsverlangen der Klägerin betroffen, namentlich die der leiblichen Brüder, des leiblichen Vaters und der Adoptivfamilie.5' Auch ihre Rechte aus Art. 8 Abs. I EMRK könnten denen Odièvres vorgehen. Der Schutz der Privatsphäre der leiblichen sowie der Adoptivfamilie stünde dem Begehren der Klägerin entgegen. ${ }^{52}$ Odièvre habe so viele Informationen erhalten, dass sie sich über die Umstände ihrer Geburt und ihrer Abgabe durch die Mutter nunmehr eine Vorstellung machen könne. ${ }^{53}$

Im Gegensatz zur früheren französischen Regelung ermögliche das Gesetz vom Januar 2002 jederzeit die Aufdeckung der Anonymität durch die biologischen Eltern. ${ }^{54}$ Durch die Schaffung eines unabhängigen Organs, den CNAOP, habe nun auch die Klägerin die Möglichkeit, die Identität ihrer leiblichen Mutter zu erfahren. Die Kenntnis sei allein von der Zustimmung der leiblichen Mutter abhängig. Die Zustimmung sei erforderlich, um die der Klägerin entgegenstehenden Interessen der Mutter zu wahren und deren Recht auf eine ungestörte Privatsphäre zu gewährleisten.5s Der Gerichtshof billigte das in der neuen französischen Regelung vorgesehene Prinzip des Konsens als verhältnismäßig: Stimmen Mutter und Kind zu, so wird die Identität preisgegeben. Verweigert die Mutter ihre Zustimmung, geht ihr Recht auf Wahrung ihrer Anonymität dem des Kindes auf Kenntnis seiner Abstammung vor. Vor dem Hintergrund seiner besonderen historischen Entwicklung bewege sich Frankreich mit der neuen Regelung zur anonymen Geburt innerhalb des jedem Konventionsstaat zustehenden Beurteilungsspielraums. Art. 8 Abs. I EMRK sei durch die französische Regelung aus dem Jahr 2002 nicht verletzt, und auch im Einzelfall Odièvres sehe der Gerichtshof keine Verletzung der Rechte der Klägerin aus der EMRK. ${ }^{56} \mathrm{Ihr}$ Recht auf Kenntnis der eigenen Herkunft müsse hinter dem Recht auf Wahrung der Privatsphäre ihrer leiblichen Familie zurückstehen.

\section{Das abweichende Votum}

Sieben Richter und Richterinnen haben sich in einem beachtlichen abweichenden Votum von der Mehrheit des Straßburger Gerichts distanziert.57 Ihre Kritik konzentriert sich im Wesentlichen auf die vom Gerichtshof vorgenommene Verhältnismäßigkeitsprüfung. Im konkreten Fall von Pascale Odièvre gäbe es keine Anzeichen, dass die Interessen der Adoptivfamilie Odièvre dem Verlangen der Klägerin auf Kenntnis der Identität ihrer leiblichen Verwandten entgegenstünden..$^{8}$ Als problematisch bezeichnen die sieben Richter darüber hinaus die Auffassung des Gerichts, dass auch die Interessen des leiblichen Vater schwerer wiegen sollen als die der Klägerin, denn der Vater hatte im Fall Odièvres offensichtlich durch seine mangelnde Unterstützung der Mutter wesentlich zur anonymen Kindesabgabe beigetragen.

Auch zeige das Urteil methodische Schwächen. Zwar stelle das Gericht die Rechte von Mutter und Kind gegenüber, eine Abwägung hinsichtlich der Schwere des Eingriffs sei

\footnotetext{
5I EGMR, I3. 2. 2003 (Fn. I), \44.

52 EGMR, I 3. 2. 2003 (Fn. I), $\ 44$.

53 EGMR, I3. 2. 2003 (Fn. I), $\$ 48$.

54 EGMR, I3. 2. 2003 (Fn. I), $\$ 49$.

55 EGMR, I 3. 2. 2003 (Fn. I), $\$ 49$.

s6 EGMR, I3.2.2003 (Fn. I), \49; mit gleicher Begründung lehnt der Gerichtshof eine Verletzung von Art. I 4 i. V.m. Art. 8 EMRK, des Gleichberechtigungsgrundsatzes, ab, den Odièvre im Wesentlichen mit dem Verlust des Erbrechts nach der biologischen Familie begründet, vgl. Entscheidung v. I 3. 3. 2003 (Fn. I), $\int S 50 \mathrm{ff}$.

57 Gemeinsames, gegenteiliges Votum der Richter Wildhaber (Schweiz), Bratza (England), Bonello (Malta), Loucaides (Zypern), Cabral Baretto (Portugal), Pellonpää (Finnland) sowie der Richterin Tulkens (Belgien); im Folgenden zitiert als: abweichendes Votum.

58 EMRK, I3. 2. 2003 (Fn. I), \$3I.
} 
aber nicht erfolgt.59 Schließlich habe Frankreich durch die neue Gesetzgebung aus

dem Jahr 2002 zwar ein unabhängiges und pluralistisch besetztes Gremium geschaffen, diesem aber tatsächlich keine Entscheidungsbefugnis eingeräumt. Allein der Konsens zwischen Eltern und Kind über die Offenbarung der Identität ermächtige den CNAOP dazu, die entsprechenden Unterlagen weiterzugeben. Es erfolge keine Prüfung im Einzelfall, ob sich die Frau, die ihre Anonymität wahren möchte, wirklich in einer rechtfertigenden Notlage befindet und ob die Gründe zur Geheimhaltung ihrer Identität im Einzelfall tatsächlich schwerer wiegen als der Eingriff in die Rechte des Kindes. ${ }^{60}$

\section{Mögliche Auswirkungen der Entscheidung auf Deutschland}

Odièvre wird also die Identität ihrer leiblichen Familie nicht erfahren. Hat diese Entscheidung des Europäischen Gerichtshofs für Menschenrechte Auswirkungen auf die in Deutschland drängende Frage nach einer gesetzlichen Regelung über Babyklappen und anonyme Geburten?

\section{Das Recht auf Kenntnis der Abstammung im deutschen Recht}

Die Lebensgeschichte Odièvres ruft bei deutschen Juristen zunächst Befremden hervor. Eine Tradition der anonymen Geburt, die Notwendigkeit der Anerkennung des leiblichen, nichtehelichen Kindes durch seine Mutter, gibt es hierzulande nicht. Nach \ I 59 I BGB gilt als Mutter diejenige Frau, die das Kind geboren hat. \6I Abs. 2 des Personenstandsgesetzes gibt jedem Kind mit Vollendung des I6. Lebensjahres ein Recht auf Einsicht in die Personenstandsbücher. Odièvre hätte in Deutschland den Namen ihrer leiblichen Mutter erfahren müssen.

Bereits der BGB-Gesetzgeber stellte eine naturrechtliche Vorstellung von Verwandtschaft, die auf der Blutsverwandtschaft zwischen Eltern und Kind beruht, in den Vordergrund der familienrechtlichen Regelungen und wandte sich bewusst von dem französischen Vorbild des Anerkennungssystems ab. ${ }^{61} \mathrm{Da}$ die biologische Mutter nach deutschem Recht mit der Geburt des Kindes feststeht, ist die Frage nach der Kenntnis der eigenen Herkunft in Deutschland nahezu ausschließlich mit der Frage nach dem leiblichen Vater und den Voraussetzungen einer Vaterschaftsklage verknüpft. ${ }^{62}$

Während der Zeit des Nationalsozialismus erfuhr diese »blutmäßige Verwandtschaft « eine neue Bedeutung. Sie konnte aufgrund der pervertierten Rassenideologie über Leben und Tod des Einzelnen entscheiden und hatte zahlreiche Regelungen zur Folge, die die Feststellung der leiblichen Vorfahren erleichtern sollten. Die Feststellung der Blutsverwandtschaft wurde zur Aussonderung »nicht-arischer« Menschen missbraucht, die biologische Abstammung der Privatsphäre des Einzelnen enthoben und zum Allgemeininteresse erklärt.

Nach der Zerschlagung des NS-Regimes mussten sich Juristen mit der Frage auseinandersetzen, ob Vaterschaftsfestellungen und Statusklagen nicht letztlich eine Fortführung nationalsozialistischer Ideologie bedeuten. Die Abkehr von der natio-

59 Abweichendes Votum (Fn. 57), \II.

60 Abweichendes Votum (Fn. 57), \I8ff. und $\$ 20$.

6I Vgl. Motive zu dem Entwurfe eines Bürgerlichen Gesetzbuches für das Deutsche Reich, Band IV, I888, S. 854,856 .

62 Vgl. Scheiwe, Babyklappe, ZRP 2001, 372. 
nalsozialistischen Ideologie führte jedoch letztlich nicht zu einer Abschaffung der Statusklage, sondern zur Aufwertung der Rechte nichtehelicher Kinder, indem das Recht auf Kenntnis der biologischen Abstammung wieder als ein Individualinteresse des nichtehelichen Kindes festgeschrieben wurde. ${ }^{63}$

Das Bundesverfassungsgericht erkannte in seinen frühen Entscheidungen bis in die 8 oer Jahre bereits das Recht des Kindes auf Kenntnis seiner Herkunft an, ließ es jedoch im Einzelfall hinter dem Interesse eines ungestörten Familienfriedens und einer frühzeitigen Klärung der familienrechtlichen Stellung des Kindes zurücktreten. ${ }^{64}$ Mit seiner Entscheidung aus dem Jahr 1989 über die Verfassungsmäßigkeit der Vorschriften über die Vaterschaftsklage bestätigte das Bundesverfassungsgericht den Verfassungsrang des »Rechts auf Kenntnis der Abstammung « als Teil des Persönlichkeitsrechts aus Art. 2 Abs. I i. V.m. I Abs. I GG. Das Bundesverfassungsgericht stellte in dieser Entscheidung fest: »Als Individualisierungsmerkmal gehört die Abstammung zur Persönlichkeit, und die Kenntnis der Herkunft bietet dem Einzelnen unabhängig vom Ausmaß wissenschaftlicher Ergebnisse wichtige Anknüpfungspunkte für das Verständnis und die Entfaltung der eigenen Individualität. Daher umfasst das Persönlichkeitsrecht auch die Kenntnis der eigenen Abstammung. «"s In späteren Entscheidungen bestätigte das Gericht diesen Grundsatz. ${ }^{66}$ Dem Staat oblag fortan nicht nur eine Schutzpflicht für das Leben des Kindes, sondern auch die Gewährleistung einer ungestörten Ausübung des Rechts auf Kenntnis seiner Abstammung. ${ }^{67}$ Der verfassungsrechtliche Auftrag hierzulande muss also lauten: Leben des Kindes und das Recht auf Kenntnis seiner Herkunft schützen.

\section{Babyklappen und anonyme Geburten in Deutschland}

In Hamburg wurde im Jahr 2000 die erste Babyklappe eröffnet, und inzwischen verfügt fast jede deutsche Region über ähnliche Einrichtungen. Anonyme Geburten werden in verschiedenen Krankenhäusern angeboten. ${ }^{68}$ Trotzdem kann die heutige Rechtslage in Deutschland nicht mit den Grundrechten der Europäischen Menschenrechtskonvention in Konflikt kommen, weil das geltende Recht hierzulande die Anonymität nicht kennt. Dass Babyklappen und anonyme Geburten gegen heute geltendes Zivil-, Straf- und Verfassungsrecht verstoßen, ist in den letzten Jahren in der juristischen Literatur immer wieder betont worden. ${ }^{69}$ Nicht zuletzt ein vom Landesjugendamt Berlin (im vergangenen Jahr) eingeholtes Gutachten zeigt, dass die bestehenden Babyklappen bei Anwendung des geltenden Rechts sofort geschlossen werden müssten. ${ }^{70}$

63 Vgl. BGH v. I8. 5. I953, NJW i953, S. I 445 f.; Neumann-Duesberg, Die Abstammungs-Feststellungsklage, NJW i950, S. I4 ff.; Schwab, Die neueste Rechtsprechung des Bundesgerichtshofs zur Abstammungsklage, NJW i956, S. $649 \mathrm{ff}$.

64 BVerfG v. 4. I2. 1974, NJW I975, S. 203 f.

65 BVerfGE 79, 256 (269), vgl. Giesen, Genetische Abstammung und Recht, JZ 1989, S. 364 (370).

66 Vgl. BVerfGE 90, 263 (270f.); BVerfGE 96, 56 (64f.).

67 Nach der neueren Entscheidung des BVerfG gibt Art. 2 I i. V. m. I I GG kein Recht auf Verschaffung der Informationen sondern lediglich ein Recht auf Schutz vor Vorenthaltung erlangbarer Informationen, vgl. BVerfGE 96, 56 (65); vgl. di Fabio zu Art. 2 I GG, in: Maunz-Düring, Grundgesetz, Kommentar, Art. 2 I GG, Rn. 2 I 2.

$68 \mathrm{Vgl}$. Verzeichnis aller Babyklappen unter www.adoption.de/info_babyklappe.html.

69 Vgl. Benöhr, Susanne/Muth, Iris A., Babyklappe und Anonyme Geburt, KJ 200I, S. 406-424, S. 408 ff; Hepting, Reinhard, »Babyklappe« und »anonyme Geburt«, FamRZ 200I, S. I 573-I 58 5; Neuheuser, Stephan, Begründet die Weggabe eines Neugeborenen in eine »Babyklappe« den Anfangsverdacht einer Straftat?, NStZ 200I, S. I75-178; Scheiwe, Kirsten, Babyklappe, ZRP 200I, S. 368-374; Wolf, Alfred, Babyklappe und anonyme Geburt - Fragen zu einer neuen Entwicklung, FRP 2001, S. 345-353; ders., Über Konsequenzen aus den gescheiterten Versuchen, Babyklappen und »anonyme " Geburten durch Gesetz zu legalisieren (im Folgenden: Wolf, Konsequenzen) FRP 2003, I I 2-I 20.

70 Wolf, Alfred, Juristische Stellungnahme zu Babyklappen und anonymen Geburten vom I 4. I 2. 2002. 
der anonymen Geburt und hätten wesentliche Änderungen des Bürgerlichen Gesetzbuches, des Personenstandsgesetzes, des Rechts über die Staatsbürgerschaft sowie des Gesetzes über die Freiwillige Gerichtsbarkeit zur Folge gehabt. ${ }^{\text {II }}$ Sie wurden eingestellt. Anhörungen von Experten hatten ergeben, dass für die bestehenden Probleme bisher kein ausreichender Lösungsansatz gefunden werden konnte. ${ }^{72}$ Mit den verfassungsrechtlichen und familienrechtlichen Bestimmungen ist die Institution der anonymen Geburt nicht vereinbar. ${ }^{73}$

Die Organisation »terre des hommes « veranstaltete im Mai 2003 eine interdisziplinäre Fachtagung zum Thema »Babyklappe und anonyme Geburt - ohne Alternative? «. ${ }^{74}$ Dabei stellte sich heraus, dass in Deutschland die Zahl der Kindestötungen und Kindesaussetzungen seit der Einrichtung von Babyklappen und anonymer Geburt nicht zurückgegangen ist. Nach wie vor werden hierzulande zwischen 30 und 35 Kinder im Jahr ausgesetzt; etwa die Hälfte von ihnen überleben. ${ }^{55}$ Mediziner, Psychologen und Soziologen bezweifeln, dass Babyklappen und anonyme Geburten ein geeignetes Mittel darstellen, um Kindestötung und Kindesaussetzung zu verhindern. Untersuchungen haben ergeben, dass für Eltern, die aus Not oder Verzweiflung ihre Kinder töten, diese Einrichtungen gerade keine Alternative bieten. ${ }^{76}$ Da Babyklappen aber tatsächlich genutzt werden, ist zu befürchten, dass sie für Frauen in Konfliktsituation eine nicht beabsichtigte Alternative zu dem als umständlich empfundenen Adoptionsverfahren darstellen. ${ }^{77}$ Gerade für diese Frauen oder Eltern ist das Angebot der anonymen Kindesabgabe nicht vorgesehen. Erziehungswissenschaftler und Psychologen weisen auf die weitreichenden Folgen der Anonymität für Kinder und abgebende Mütter hin, denn sowohl bei den Kindern als auch bei den abgebenden Müttern kann die anonyme und damit endgültige Trennung zu schweren psychischen Erkrankungen führen..$^{8}$

Das Urteil des Europäischen Gerichtshofs vom Februar 2003 hat auch in Frankreich nicht zu einem Abbruch der Diskussion über die anonyme Geburt geführt. Welche Schwierigkeiten die französische Regelung über die anonyme Geburt verursacht, zeigt der Fall des heute dreijährigen Benjamin Peter, der zur Zeit Frankreichs Öffentlichkeit und Gerichte beschäftigt.

Benjamin wurde im Mai 2000 anonym geboren. Noch vor seiner Geburt hatte ihn sein leiblicher Vater anerkannt. Benjamins Eltern haben sich vor seiner Geburt getrennt, die Mutter hat ihn daraufhin anonym entbunden. Benjamin ist nach seiner Geburt den

7I Gesetzesentwurf zur Änderung des Personenstandsgesetzes der CDU/CSU-Fraktion v. I 2. I0. 2000, BTDrs. I4/4425; Interfraktioneller Entwurf eines Gesetzes zur Regelung anonymer Geburten v. 23. 4. 2002, BT-Drs. I 4/88 56; Entwurf eines Gesetzes zur Regelung anonymer Geburten des Landes Baden-Württemberg aus dem Jahr 2002, BR-Drs. 506/02; vgl. Wolf, FRP 2003, S. I I 2-I 20.

72 Vgl. ausführliche Darstellung bei Wolf, FRP 2003, S. I I ff. m. w. N.

73 Riedel, Ulrike, Erinnerung an das geltende Recht, in: terre des hommes, Babyklappe (Fn. 74), S. 33 ff.; Wolf, Alfred, Familienrechtliche Überlegungen, in: terre des hommes, Babyklappe (Fn. 74), S. $56 \mathrm{ff}$.

74 Die Diskussionsbeiträge und Vorträge der Tagung sind zusammengestellt in: terre des hommes (Hrsg.), Babyklappe und anonyme Geburt - ohne Alternative, Osnabrück 2003; siehe auch: www.tdh.de.

75 Vgl. Bott, Regula/Swientek, Christine, Kindestötung und Kindesaussetzung in Zeiten von Babyklappe und anonymer Geburt, in: terre des hommes, Babyklappe (Fn. 74), S. I68 ff.

76 Rohde, A./Raic, D./Varchim-Schultheiß, K./Marneros, A., Infanticide: Sociobiographical background and motivational aspects, Archives of Women's Mental Health, I998, S. I $25-130$; Rohde, Anke, Welche Mütter töten ihre Kinder?, in: terre des hommes, Babyklappe (Fn. 74), S. I 3 I ff.

77 Vgl. Herpich-Behrens, Ulrike, LJA Berlin, Was brauchen Mütter in höchster Not wirklich?, in: terre des hommes, Babyklappe (Fn. 74), S. I 47 ff.; Wacker, Bernd, Gefahr des Kinderhandels, in: terre des hommes, Babyklappe (Fn. 74), S. $86 \mathrm{ff}$.

78 Bundesarbeitsgemeinschaft Adoptierter-BARGEA, Stellungnahme, in: terre des hommes, Babyklappe (Fn. 74), S. I 7 ff.; Bott, Regula, Wunsch und Wirklichkeit, in: terre des hommes, Babyklappe (Fn. 74), S. I 3 ff.; Swientek, Christine, Lebenszeitfolgen bei Müttern, die ihre Kinder abgeben, in: terre des hommes, Babyklappe (Fn. 74), S. I 20 ff. 
staatlichen Stellen, im Oktober 2000 dann dem Ehepaar Faux übergeben worden, das sich seit drei Jahren intensiv um das zunächst sehr kranke Kind gekümmert hat und nun die in Aussicht gestellte Adoption verlangt. Doch auch der leibliche Vater möchte mit Benjamin leben. Er begab sich nach dem errechneten Geburtstermin auf die Suche nach dem Sohn, den er jedoch erst Monate nach der Geburt fand. Zu dieser Zeit lebte Benjamin bereits bei seinen Adoptiveltern. Seine leiblichen Eltern kennt er nicht. Am I6. 5.2003 hat das »tribunal de grande instance de Nancy « Benjamin dennoch seinem leiblichen Vater zugesprochen. ${ }^{79}$

Zwar schließt Art. 353 C. C. ab dem Zeitpunkt der Freigabe des Kindes zur Adoption eine Wiederherstellung des Verwandtschaftsverhältnisses zur leiblichen Familie grundsätzlich aus, doch gilt dies nach Ansicht des Gerichts in Nancy nur für eine Anerkennung des Kindes durch seine Eltern nach der Geburt. Der Vater hatte Benjamin aber schon vor der Geburt anerkannt. Er habe daher, so das Gericht in Nancy, ein Recht darauf, seinen Sohn zu versorgen und zu erziehen.

Die Frage der Auslegung des Art. 353 C. C. ist unter französischen Juristen umstritten. Nach einer anderen Ansicht bleibt eine Anerkennung eines anonym geborenen Kindes durch seinen nichtehelichen Vater auch vor der Geburt des Kindes ausgeschlossen, weil die anonym gebärende Mutter aus juristischer Sicht nie Mutter werden könne und die Anerkennung eines Kindes durch den Vater zunächst überhaupt eine Mutterschaft voraussetze. ${ }^{8}$ Kein Vater ohne Mutter. Diese Ansicht verfolgt zwar konsequent den Gedanken der Anonymität weiter, führt jedoch zu einer unerträglichen Benachteiligung des nichtehelichen, leiblichen Vaters. ${ }^{8}{ }^{8}$ Es bleibt abzuwarten, welche Entscheidung das Gericht nächster Instanz im Fall Benjamin Peter treffen wird. Das Beispiel Benjamin zeigt überdeutlich, zu welchen Problemen die französische Regelung über anonyme Geburten führt. Benjamin Peter hat einen leiblichen Vater, eine leibliche Mutter, die das Kind nach der Zurückweisung des Adoptionsverlangens der Pflegeeltern nach Art. 352 C. C. wieder anerkennen könnte, und Eltern, bei denen er seit drei Jahren lebt und die für den Dreijährigen seine engsten Bezugspersonen sind. Pierre Verdier, Leiter der wohl wichtigsten, französischen Organisation zur Wahrung des Rechts auf Abstammung, ${ }^{82}$ schreibt in der französischen Tageszeitung Libération sinngemäß: Sie alle sind Opfer der französischen Gesetzgebung über die anonyme Geburt und der ihr zugrunde liegenden Fiktion, dass ein Kind, das anonym geboren wird, tatsächlich keine leiblichen Eltern habe. ${ }^{83}$

Im deutschen Recht und seiner Tradition sind das Recht auf Kenntnis der Abstammung und die Rechte des nichtehelichen Vaters, zuletzt bestätigt in einer Entscheidung des Bundesverfassungsgerichts vom Mai 2003, ${ }^{84}$ Teil der Verfassungsordnung. Die Anonymität der Eltern ist dem deutschen Rechtssystem fremd. Die Legalisierung von Babyklappen wird auch in Deutschland offensichtlich von niemandem mehr angestrebt. Ihre Einrichtung bleibt rechtsmissbräuchlich. Ebenso rechtsmissbräuchlich ist die Praxis der »anonymen Geburt«. Wer die familien- und verfassungsrechtlichen Grundsätze ernst nimmt, kann und wird sie nicht legalisieren.

79 Des Deserts, Sophie, Parents de cœur, parents de sang, in: Le Nouvel Observateur, 19.6. 2003; Pereira, Acacio, Un enfant né sous $\mathrm{X}$ restitué à son père biologique, in: le monde, 23.5. 2003.

80 Rubellin-Dechivi, Jacqueline, La filiation de l'enfant fait l'objet d'une reconnaissance prénatale puis né sous X: JCP 2003 , no. 27 , p. I 254.

8I Zur Problematik der Ausgrenzung des nichtehelichen Vaters im Falle der Legalisierung anonymer oder geschützer Geburten siehe auch : Wolf, Alfred, Juristische Bemerkungen zur vertraulichen Geburt v. I0. 9. 2003, www.oneworldweb.de/tdh/themen/babyklappe/vg-_jur_bemerkungen.html.

82 Pierre Verdier ist Präsident der «Coordination des actions pour le droit à la connaissance des origines ».

83 Vgl. Verdier, Pierre, a qui appartient un enfant?, in: Libération v. 24.6.03.

84 BVerfG I BvR I493/96 v. 9. 4. 2003, http://www.bverfg.de/entscheidungen/rs200340-rbvri 49396.html. 